\title{
TIRSPEC: TIFR Near Infrared Spectrometer and Imager
}

\author{
J. P. Ninan*, , D. K. Ojha*, S. K. Ghosh*,†, S. L. A. D'Costa*, M. B. Naik*, \\ S. S. Poojary*, P. R. Sandimani*, G. S. Meshram*, R. B. Jadhav*, \\ S. B. Bhagat*, S. M. Gharat*, C. B. Bakalkar*, T. P. Prabhu ${ }^{\ddagger}$, \\ G. C. Anupama and D. W. Toomey ${ }^{\S}$ \\ * Tata Institute of Fundamental Research \\ Homi Bhabha Road, Colaba, Mumbai 400 005, India \\ ${ }^{\dagger}$ National Centre for Radio Astrophysics \\ Tata Institute of Fundamental Research \\ Pune 411 007, India \\ ${ }^{\ddagger}$ Indian Institute of Astrophysics \\ Koramangala, Bangalore 560 034, India \\ $\S$ Mauna Kea Infrared, LLC \\ 21 Pookela St. Hilo, HI 96720, USA \\ 『ninan@tifr.res.in
}

Received 2014 June 27; Accepted 2014 August 14; Published 2014 November 17

\begin{abstract}
We describe the TIFR Near Infrared Spectrometer and Imager (TIRSPEC) designed and built in collaboration with M/s. Mauna Kea Infrared LLC, Hawaii, USA, now in operation on the side port of the 2-m Himalayan Chandra Telescope (HCT), Hanle (Ladakh), India at an altitude of 4500 meters above mean sea level (amsl). The TIRSPEC provides for various modes of operation which include photometry with broad and narrow band filters, spectrometry in single order mode with long slits of $300^{\prime \prime}$ length and different widths, with order sorter filters in the Y, J, H and K bands and a grism as the dispersing element as well as a cross dispersed mode to give a coverage of 1.0 to $2.5 \mu \mathrm{m}$ at a resolving power $R$ of $\sim 1200$. The TIRSPEC uses a Teledyne $1024 \times 1024$ pixel Hawaii- 1 PACE array detector with a cutoff wavelength of $2.5 \mu \mathrm{m}$ and on HCT, provides a field of view of $307^{\prime \prime} \times 307^{\prime \prime}$ with a plate scale of $0.3^{\prime \prime} /$ pixel. The TIRSPEC was successfully commissioned in June 2013 and the subsequent characterization and astronomical observations are presented here. The TIRSPEC has been made available to the worldwide astronomical community for science observations from May 2014.
\end{abstract}

Keywords: Near infrared astronomy, spectrometer, imager.

\section{Introduction}

Near infrared astronomy (NIR) using medium sized $(2-4 \mathrm{~m})$ telescopes has greatly opened up the study of many astrophysically cool objects like evolved giants and supergiants, low mass stars, red and brown dwarfs and Galactic star-forming regions. In the recent past there have been many developments in the field of NIR detector materials and arrays which have brought NIR astronomical observations almost on par with the optical observations. Within the Indian astronomical scene, a few NIR observational facilities exist viz., Indian

${ }^{\top}$ Corresponding author.
Institute of Astrophysics (IIA) NIRCAM on the 2-m Himalayan Chandra Telescope (HCT), Hanle (Ladakh) and the Physical Research Laboratory's (PRL) NICMOS III camera on the 1.2-m PRL telescope at Mount Abu. Recently, Tata Institute of Fundamental Research (TIFR) has also developed an NIR camera (1.0 to $3.7 \mu \mathrm{m})$ (Ojha et al., 2012a; Naik et al., 2012; Ninan et al., 2013a) based on the Raytheon InSb $512 \times 512$ pixel Focal Plane Array (Aladdin III Quadrant) which is currently being used with the 2-m Inter-University Centre for Astronomy and Astrophysics's Girawali Observatory telescope, near Pune. Besides this, TIFR has also been observing in the far infrared (FIR) band 
(120 to $220 \mu \mathrm{m})$ using the TIFR 1-m FIR balloon borne telescope (Ghosh et al., 1988; Ghosh, 2010). To complement these studies in the NIR band, the need was felt for a NIR spectrometer in the 1.0 to $2.5-\mu \mathrm{m}$ range, which could be used with one of the 2 -m class telescopes available in India. To meet this need, the TIFR Near Infrared Spectrometer and Imager (TIRSPEC) (Ojha et al., 2012b) was conceived, to provide spectrometry in the 1.0 to $2.5-\mu \mathrm{m}$ band with a medium spectral resolving power of $\sim 1200$ to be used on one of the side ports of the $2-\mathrm{m}$ HCT at Hanle (Ladakh) (4500 m amsl), along with the existing optical Himalaya Faint Object Spectrograph and Camera on the main port, to allow for near simultaneous observations in the optical and NIR bands.

TIRSPEC was developed in collaboration with M/s. Mauna Kea Infrared, LLC (MKIR), Hawaii, USA, to provide both imaging as well as spectroscopic capabilities in the 1.0 to $2.5-\mu \mathrm{m}$ NIR wavelength range and was designed around a Teledyne $1024 \times 1024$ pixel Hawaii- 1 PACE array detector as the focal plane array. When mounted on the side port of the 2-m HCT at Hanle (Ladakh), the field of view (FoV) in the imaging mode is $307^{\prime \prime} \times 307^{\prime \prime}$ with a plate scale of $0.3^{\prime \prime} /$ pixel, while in the spectroscopic mode it covers a wavelength range from 1.0 to $2.5 \mu \mathrm{m}$ with a spectral resolving power of $\sim 1200$. The first light for this instrument on the HCT was observed on June 21, 2013 and after the characterization and engineering observational runs, the TIRSPEC was released to the worldwide astronomical community for science observations from May 1, 2014.

In this paper, we describe the technical details of TIRSPEC (Sec. 2), and also the characterization and performance analysis done before releasing the instrument to the users for scientific observations (Sec. 3). We also present the performance analysis of TIRSPEC as related to the Hanle telescope site and some samples of science observations conducted during the engineering runs (Sec. 4). The data reduction tool developed for TIRSPEC is explained in Sec. 5 and we conclude with the present status and some of the planned upgrades which are to be carried out in the near future.

\section{Instrument Overview}

\subsection{Structure}

TIRSPEC has an overall envelope size of $481 \mathrm{~mm}$ (width) $\times 600 \mathrm{~mm}($ length $) \times 500 \mathrm{~mm}$ (height) The

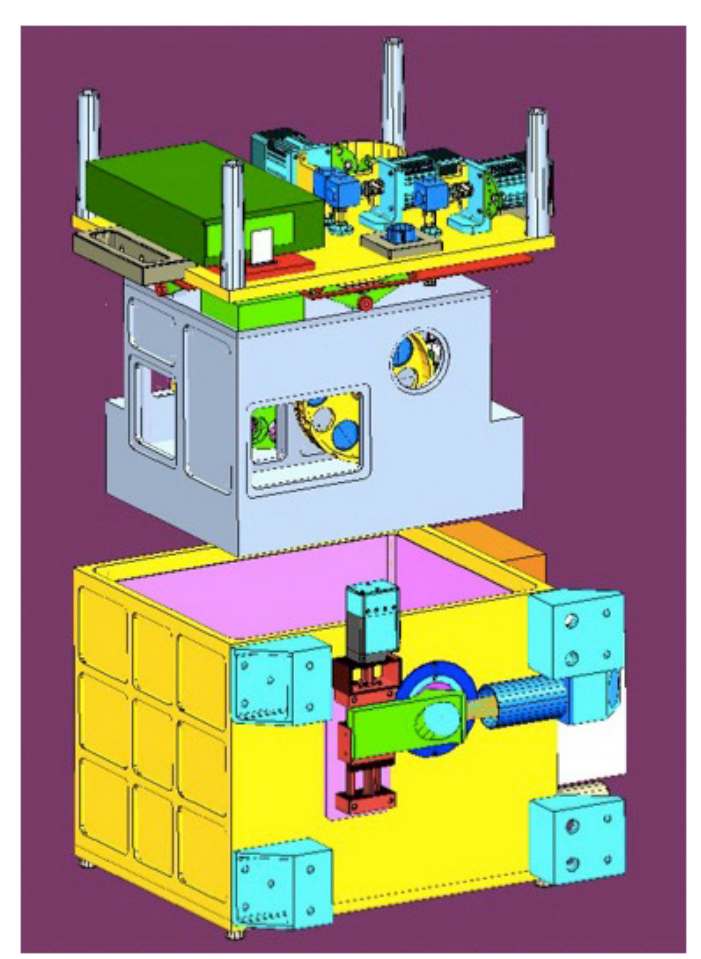

Fig. 1. (Color online) Exploded front view of the TIRSPEC cryostat. The yellow tub and top plate are the outer vacuum jacket. The pink color shields are floating radiation shields. The grey enclosure is the LN2 cooled enclosure which houses the optics.

cryostat is designed as a double bath-tub which allows all the vacuum penetrations to be from the top plate of the cryostat. Figure 1 shows an exploded view of the cryostat with the outer cube providing the vacuum jacket. The optical bench is mounted within the liquid nitrogen (LN2) cooled enclosure which hangs inside the outer vacuum jacket from 4 rigid fiber-glass $\mathrm{V}$-trusses connected to the top plate. Thermally insulated mirror finished floating shields line the inner side of the vacuum jacket to provide good radiation shielding and to improve LN2 hold time. The LN2 tank can hold about 8.6 liters of LN2 which provides a hold time of more than 36 hours at the observatory site. The array controller electronics and the power supply for the motors and electronics are mounted on one side of the cryostat. The three motors for the slit wheel and the two filter wheels are mounted on the outer side of the top plate and are coupled to the filter and slit wheels through right-angled gears and ferrofluidic vacuum feed-throughs. A calibration lamp assembly provides for spectral line calibration of the spectrometer using an argon lamp with a movable plane mirror which can be moved 
in and out of the optical path using an Animatics SmartMotor and linear stage arrangement. The calibration box also hosts a tungsten lamp for continuum flats with an integrating sphere.

\subsection{Optics}

TIRSPEC's optics were designed to be used on the side port of the $2-\mathrm{m} \mathrm{HCT}$, to provide an image scale of $0.3^{\prime \prime} /$ pixel covering a FoV of $307^{\prime \prime} \times 307^{\prime \prime}$. Figure 2 shows the layout of the optics. The f/9.2 beam from $\mathrm{HCT}$ enters the instrument via the $\mathrm{CaF}_{2}$ window. At the telescope focal plane (TFP) there

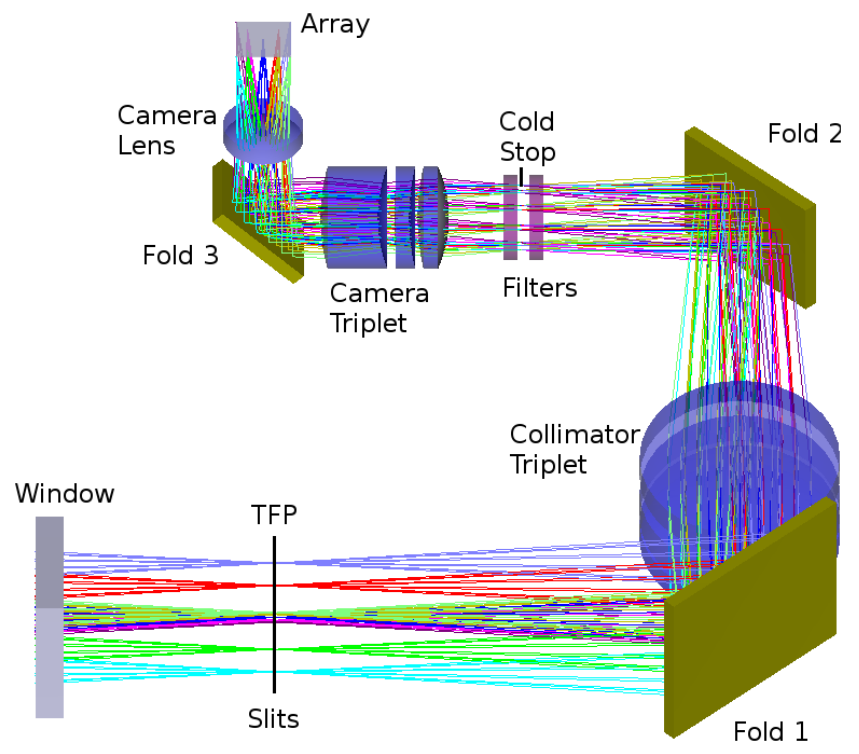

Fig. 2. TIRSPEC's folded optics design. is a 11-position slit wheel which has 5 short slits (10" length) and 5 long slits (300" length) with slit widths of $0.9^{\prime \prime}, 1.48^{\prime \prime}, 1.97^{\prime \prime}, 2.96^{\prime \prime}$ and $7.92^{\prime \prime}$, as well as one open position for the imaging mode. A collimator system consisting of a $\mathrm{BaF}_{2}-\mathrm{LiF}-\mathrm{BaF}_{2}$ lens triplet is used to obtain a good quality achromatic image of the telescope aperture at the cold stop which is sandwiched between two 12-position filter wheels. The first filter wheel has broad band filters $\left(\mathrm{J}, \mathrm{H}, \mathrm{K}_{\mathrm{s}}\right)$ and order sorter filters $(\mathrm{Y}, \mathrm{J}, \mathrm{H}, \mathrm{K})$ and two cross dispersing grisms (HK and YJ). The second filter wheel has seven narrow band filters, one grism, block for taking dark images and an open position. Table 1 lists the details of imaging filters and Fig. 3 shows the transmission curves of all the available imaging and order sorter filters.

The collimated beam is then imaged on to the focal plane array detector using a $\mathrm{BaF}_{2}-\mathrm{LiF}-\mathrm{ZnSe}$

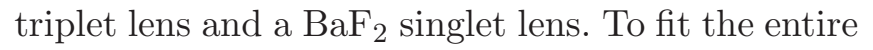

Table 1. Imaging filters.

\begin{tabular}{lc}
\hline Filter & Band pass $(\mu \mathrm{m})$ \\
\hline $\mathrm{J}$ & $1.17-1.33$ \\
$\mathrm{H}$ & $1.49-1.78$ \\
$\mathrm{~K}_{\mathrm{S}}$ & $1.99-2.30$ \\
Methane off & $1.584(3.6 \%)$ \\
[Fe II] & $1.645(1.6 \%)$ \\
Methane on & $1.654(4.0 \%)$ \\
$\mathrm{H} 2(1-0) \mathrm{S}(1)$ & $2.1239(2.0 \%)$ \\
Br $\gamma$ & $2.166(0.98 \%)$ \\
$\mathrm{K}-\mathrm{Cont}$ & $2.273(1.73 \%)$ \\
$\mathrm{CO}(2-0)$ & $2.287(1.33 \%)$ \\
\hline
\end{tabular}

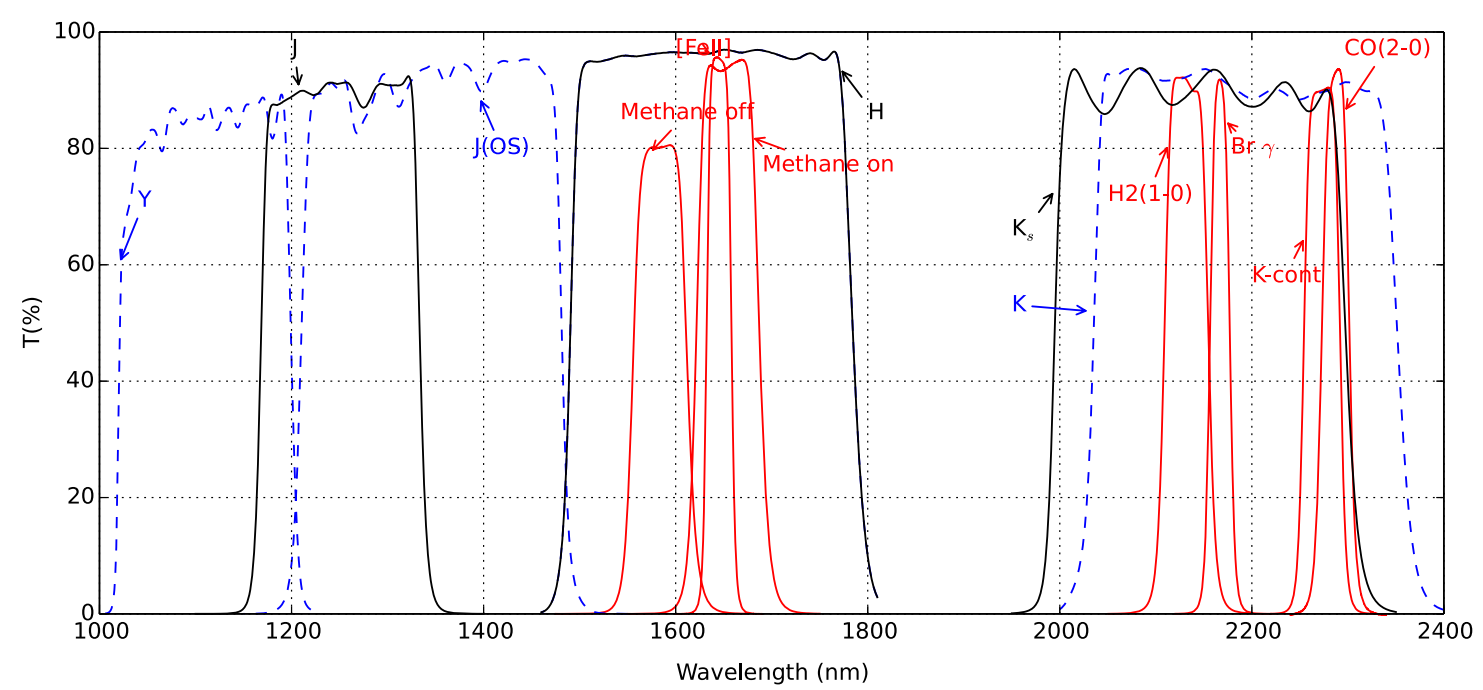

Fig. 3. (Color online) Transmittance curves of various filters on TIRSPEC. The black curves are the broad band J, H and $\mathrm{K}_{\mathrm{s}}$ filters, the red curves are the narrow bands, and the blue dashed curves are the order sorters for spectroscopy. 
optics inside the LN2 cooled enclosure, the optical path is folded at three locations using gold coated fused silica plane mirrors.

The focal plane array used in TIRSPEC is a HAWAII-1 PACE Array, ${ }^{(a)}$ manufactured by Teledyne Scientific \& Imaging, LLC, USA. It is a $1024 \times 1024$ array, with a pixel size of $18 \mu \mathrm{m}$ square and has a cutoff wavelength of $2.5 \mu \mathrm{m}$. The array has $\mathrm{HgCdTe}$ detector layer on top and a silicon readout layer below which contains readout amplifiers and associated circuits. Both the layers are connected by indium bumps at each pixel (Hodapp et al., 1996; Rieke, 2007). The array has four quadrants which are read out simultaneously. Readout starts from the bottom left corner pixel to the top right corner pixel of each quadrant at a time of $3 \mu \mathrm{s} /$ pixel. The detector is operated at a temperature of $76-77 \mathrm{~K}$ at Hanle (Ladakh).

Spectropscopy using TIRSPEC can be done using the single order mode or the cross dispersed mode. In both modes, a YJHK grism is positioned in the second filter wheel. This grism has a groove width of $12.4 \mu \mathrm{m}$ and a prism angle of $21.9^{\circ}$ which disperses $\mathrm{Y}, \mathrm{J}, \mathrm{H}$ and $\mathrm{K}$ in its 6th, 5th, 4th and 3rd orders respectively. To obtain the spectrum of each order separately, in the single order mode, the corresponding order sorter filter is positioned in the first filter wheel which blocks the other orders.

In the cross dispersed mode, we can simultaneously obtain spectra in two orders. Two modes are provided which cover the $\mathrm{Y}$ and $\mathrm{J}$ windows or the $\mathrm{H}$ and $\mathrm{K}$ windows. This is accomplished by positioning the YJHK grism in one filter wheel and a cross dispersing grism in the second wheel. The cross dispersing grism is mounted 90 degrees rotated with respect to the YJHK grism and has a custom order sorter filter that covers both orders. The first order dispersion of the YJ cross dispersed grism with groove width of $6.2 \mu \mathrm{m}$ and prism angle of $8^{\circ}$ is used to obtain cross dispersed $\mathrm{Y}$ and $\mathrm{J}$ spectrum. Similarly, the first order dispersion of the HK cross dispersed grism with groove width of $15.4 \mu \mathrm{m}$ and prism angle of $5^{\circ}$ is used to obtain cross dispersed $\mathrm{H}$ and $\mathrm{K}$ spectrum. All the grisms are directly ruled grisms. Table 2 shows the wavelengths covered in each mode.

${ }^{\mathrm{a}} \mathrm{HgCdTe}$ Astronomy Wide Area Infrared Imager -1 , Producible Alternative to CdTe for Epitaxy.
Table 2. Wavelength coverage in different spectroscopy modes.

\begin{tabular}{lc}
\hline Spectroscopy order & Wavelength range $(\mu \mathrm{m})$ \\
\hline $\mathrm{Y}$ & $1.02-1.20$ \\
$\mathrm{~J}$ & $1.21-1.48$ \\
$\mathrm{H}$ & $1.49-1.78$ \\
$\mathrm{~K}$ & $2.04-2.35$ \\
$\mathrm{YJ}^{*}$ & $1.02-1.49$ \\
$\mathrm{HK}^{*}$ & $1.50-1.84 \& 1.95-2.45$ \\
\hline
\end{tabular}

*YJ and HK are cross dispersed modes.

Note: 1.84 to $1.95 \mu \mathrm{m}$ is not part of the spectrum in HK due to their dispersion being outside the physical dimensions of the detector array.

\subsection{Electronics and control systems}

The HAWAII-1 PACE array is controlled by an ARC Gen III array controller (also known as Leach Controller or SDSU Controller) from Astronomical Research Cameras, Inc. (ARC), San Diego, USA. The array is mounted on a Fanout Board inside the cryostat. The image data is sent to the ARC46: 8-Channel IR Video Processor in the array controller box mounted at the side of TIRSPEC cryostat where it is amplified and digitized. The ARC-32 CCD and IR Clock Driver Board provides the clocks to the array and the controller boards. The ARC-22: $250 \mathrm{MHz}$ Fiber Optic Timing Board, Gen III, generates the waveforms as well as communicates with a PCI (Peripheral Component Interconnect) interface board, the ARC-64: $250 \mathrm{MHz}$ PCI Interface board, Gen III, mounted in the TIRSPEC control computer, via a duplex fiber optic cable link which transfers data and commands between the computer and the array controller box. The cryostat and array temperatures are monitored using a Lakeshore temperature controller Model 335.

The slit, filter wheels and calibration box mirror are moved using independent SmartMotors manufactured by Animatics Corporation, Milpitas, USA. The motors are controlled through a standard Ethernet interface. The slit and filter wheels have a spring loaded detent mechanism with a thin film Hall sensor to detect the position of the wheel.

\subsection{Software}

The TIRSPEC instrument is controlled from the TIRSPEC control computer using the main control software. The TIRSPEC control computer is connected to two independent networks with one 


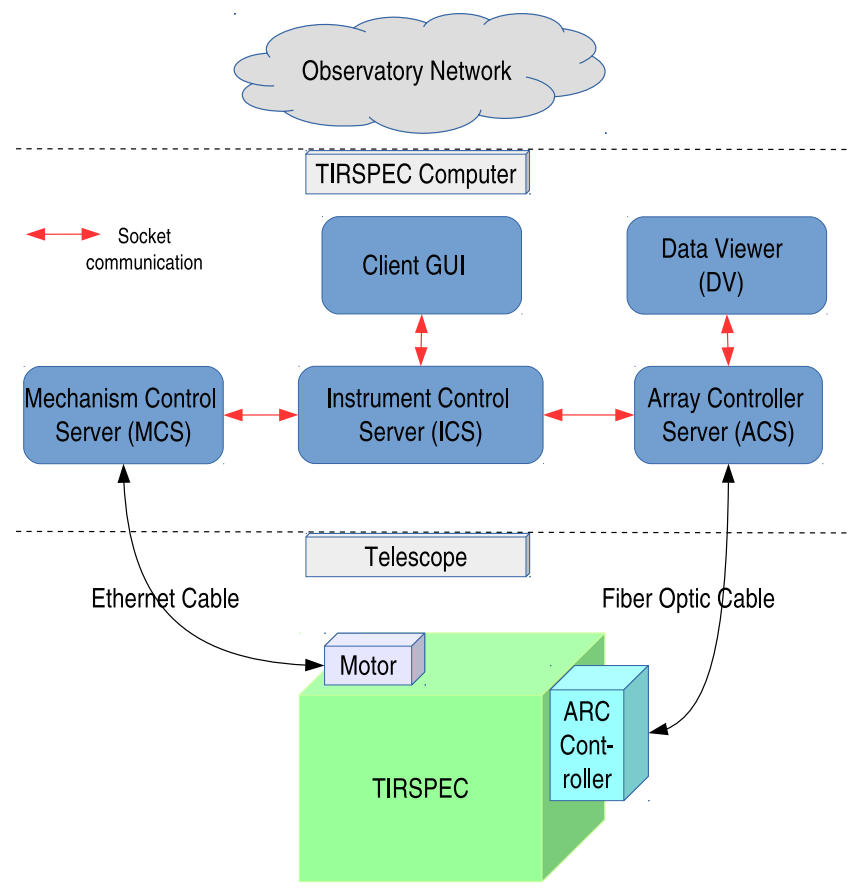

Fig. 4. Overall structure of TIRSPEC instrument control software.

being used to communicate with the observatory network for remote access, and the other being used to communicate with the TIRSPEC components which includes the Animatics SmartMotors and the TIRSPEC power control switches. The overall structure of the instrument control software is shown in Fig. 4.

The Instrument Control Server (ICS) software is the central server which controls all the other servers. It communicates with the other servers via UNIX style sockets. The ICS receives commands from the client graphical user interface (GUI) as well as returns instrument status to the GUI. When the client requests a particular filter to be in position, the ICS receives the command from the GUI and if it is a valid command, it sends the instruction to the Mechanism Control Server (MCS). The MCS talks via the Ethernet link with the corresponding Animatics SmartMotor which turns the filter wheel to the desired position. When the client requests an exposure of a specified integration time, the ICS receives the command, and if the system is ready, it sends the instruction to the Array Controller Server (ACS). The ACS talks via the ARC application programming interface (API) to the ARC controller. The ARC device drivers and API are highly customized versions of the standard code provided by Astronomical Research
Cameras, Inc. Once the CPU receives an interrupt from the PCI board after the data is fully written to the system memory, ACS generates FITS $^{(\mathrm{b})}$ image from the raw data and saves it to the hard-disk in standard FITS format. It also sends the data via a socket to the quick look viewer software DV. ${ }^{\text {(c) }} \mathrm{DV}$ has tools to do basic image arithmetic as well as quick measurements to check image quality.

\subsubsection{Readout mode and image generation}

When an exposure is not being taken, the array is continuously reset globally. At the beginning of any exposure, the reset pulse is stopped and the detector is read out non-destructively. The detector can be read out in various modes. For optimal results, the sample-up-the-ramp (SUTR) readout mode has been chosen as the default mode. The pixels are read at a time of $3 \mu$ s per pixel and since all four quadrants of the detector are readout simultaneously, it takes 0.9 seconds to complete one full readout of the array. For an integration time $I$, the actual exposure time is automatically adjusted to the nearest multiple of 0.9 second and the actual integration time used is written to the header of the data file. A total of $I / 0.9$ non-destructive readouts of the cumulative counts in the array pixels are executed during the duration of the exposure. Thus, the raw data for each exposure is a data cube with the pixels on the two axes and time on the third axis. The flux in each pixel is then calculated by fitting a slope by linear regression to this SUTR readout data cube along the time axis. Due to the reset anomaly discussed later in Sec. 3.2, one needs to subtract the dark readout cube from the image data cube before linear slope fitting. The SUTR readout technique gives us unique capabilities to recover data from cosmic ray $(\mathrm{CR})$ hits and saturation.

\subsubsection{Cosmic ray hit healing}

HCT at Hanle $(4500 \mathrm{~m}$ amsl) is prone to much higher CR hits than lower altitude observatories. Since the SUTR readout has time resolved data, the readout counts before and after a CR hit can be used to retrieve the slope of the flux at that pixel. Figure 5 shows the typical SUTR readout generally seen for a CR hit. The sudden jump in the ramp can be detected using a digital filter shown in inset of

\footnotetext{
${ }^{\mathrm{b}}$ Flexible Image Transport System.

${ }^{\mathrm{c}}$ Data Viewer by NASA Infrared Telescope Facility (IRTF), Hawaii, USA.
} 


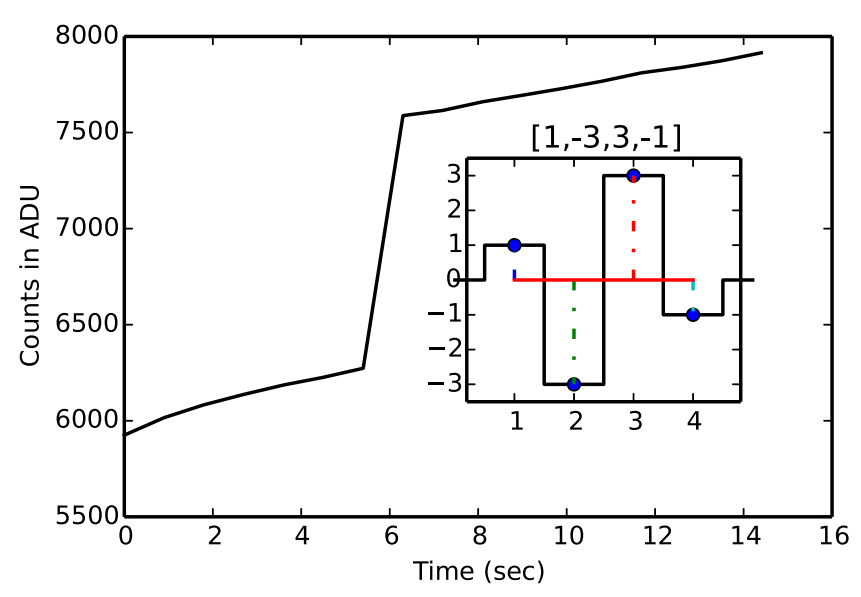

Fig. 5. CR hit between the 5th and 6th seconds during an SUTR readout exposure. The inset shows the digital filter used to detect these events.

Fig. 5. This filter is created by convolution of a first difference filter and a Mexican hat filter. After identifying the CR hit location, the slope in linear sections of the SUTR data is estimated and averaged (weighted by the error in slope of each section).

\subsubsection{Saturation healing}

Another advantage in using the SUTR readout is that saturated pixels can be selectively discarded. This is especially useful when observing fields which have both bright and faint stars. The flux is estimated using only the readouts during the linear regime of the pixels containing bright saturating stars, while for the remaining pixels, the entire data is used. This effectively increases the dynamic range of the detector beyond the actual well depth.

\subsubsection{Non-linearity correction}

From SUTR readout data we have estimated the non-linearity correction coefficients for each pixel, which can be applied to reconstruct the data beyond $1 \%$ non-linearity. This has not been done by default to any of the data. In general, all the data beyond the linear regime is discarded before fitting the slope.

\section{Characterization and Performance}

\subsection{Dark current}

Dark current noise determines the sensitivity limit we can attain in the spectroscopy mode. Unlike the imaging mode, the noise in a faint star continuum signal is dominated by dark current and effective readout noise. Dark current is measured by reading out the array cooled to $77 \mathrm{~K}$ (normal operating temperature) with the cold block filter in the second filter wheel.

The measured dark current has an exponential fall off at the beginning of the exposure. This high value at the beginning is due to the reset anomaly of the detector and is not due to high dark current. The reset anomaly is discussed in more detail in Sec. 3.2. Hence, the actual dark current of the instrument is estimated only after the detector output has stabilized. A straight line fit to the readout values after skipping the first $27 \mathrm{~s}$ of data from the start of the exposure up to $100 \mathrm{~s}$ gave a dark current value of $0.032 \mathrm{ADU} / \mathrm{s}$. This multiplied by gain (see Sec. 3.4) gives us an estimate of the dark current $\sim 0.19 \mathrm{e}^{-} / \mathrm{s}$. This value matches with estimates of dark current by others on similar HAWAII-1 PACE $1 \mathrm{k} \times 1 \mathrm{k}$ Array (Acosta-Pulido et al., 2003). Stability of dark current over a time scale of one night was tested to decide the number of dark frame readouts needed to be taken during each night. It was found that it would be enough to take a few sets of dark frame readouts at the beginning and end of each night.

\subsection{Reset anomaly}

HAWAII-1 PACE array has been known to have a non-linear behaviour immediately after the detector is reset (Finger et al., 2000; Acosta-Pulido et al., 2003). In spite of resetting at the same frequency as the readout, to reduce the effects of the reset anomaly as suggested by Riopel et al. (2004), we still see the reset anomaly in our readouts. The green curve in Fig. 6 shows the non-linear output obtained in the dark readout due to the reset anomaly. Since this is a strong function of the time that has elapsed since the reset at the beginning of exposure, we get a vertical gradient in the raw image frames in each of the quadrants as shown in Fig. 7. One solution to this anomaly is to take long exposures and discard the first few readouts. However, this cannot be done for imaging in the $\mathrm{H}$ and $\mathrm{K}$ bands or for bright stars due to saturation. Hence, it is crucial to decouple this nonlinear readout portion to obtain the true linear slope corresponding to the flux. This non-linear behavior was found to be an additive effect, and can be subtracted out to obtain the linear curve corresponding to the actual flux falling on the detector. Figure 6 shows the linear curve (dashed line) recovered after 


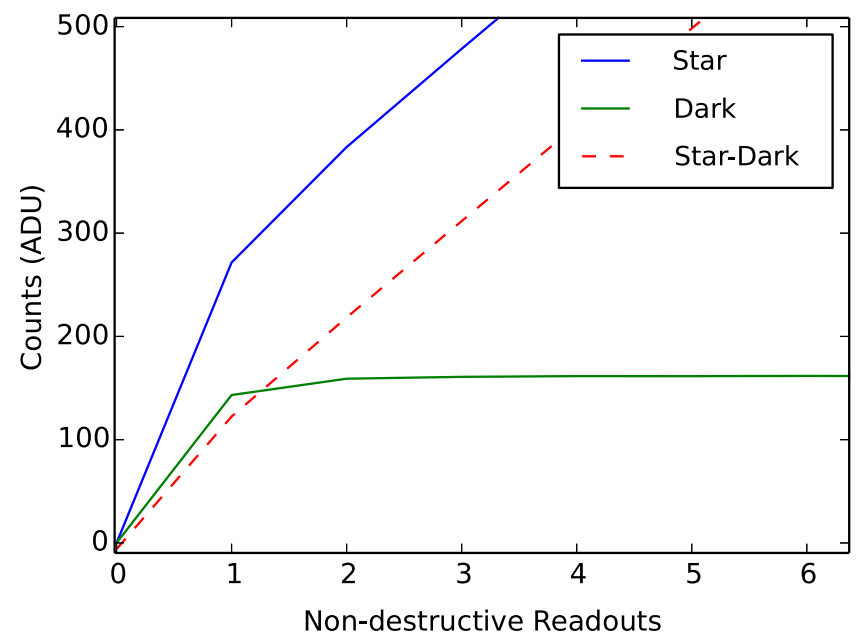

Fig. 6. (Color online) The linear slope in sample-up-theramp (SUTR) readout. Blue curve shows the readout of a pixel exposed to star and green line shows the dark readout. Red dashed line is the linear readout which is recovered after subtracting the non-linear dark in image data cube.
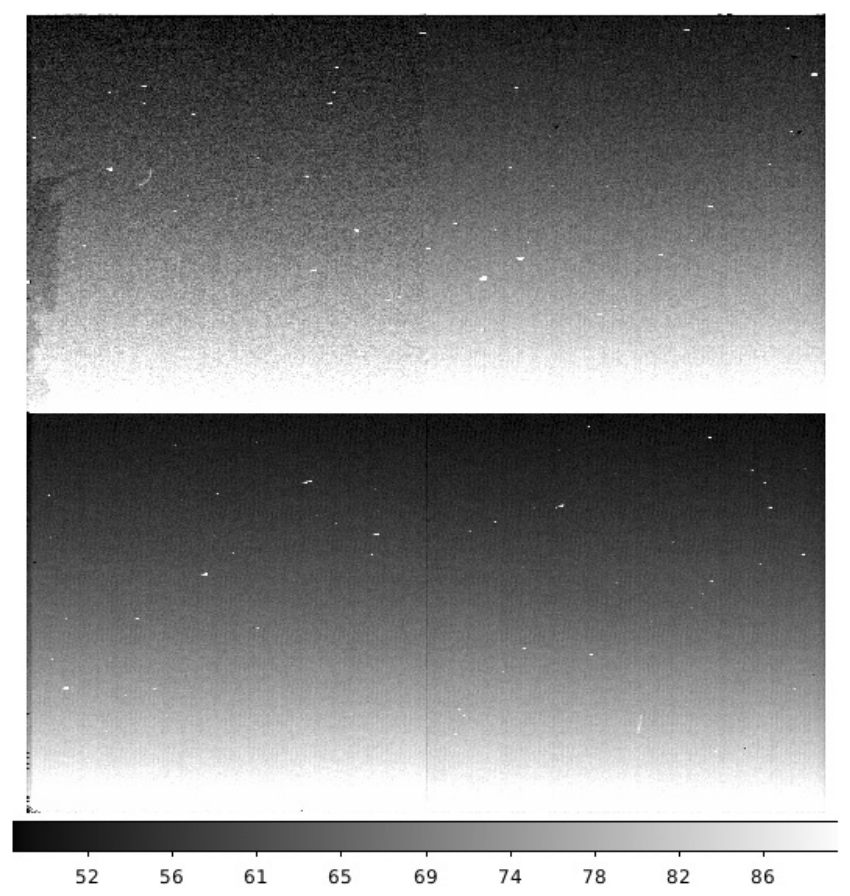

Fig. 7. The vertical gradient in each quadrant of the raw image due to the reset anomaly in a 4-s dark exposure. This gradient in the raw image can be removed fully by dark subtraction.

subtracting the dark readout (green curve). Finger et al. (2000) had also shown this additive nature of the reset anomaly.

To study the noise statistics of these large counts obtained at the initial seconds of the exposure, multiple dark SUTR readouts were taken and the dark current was plotted against the

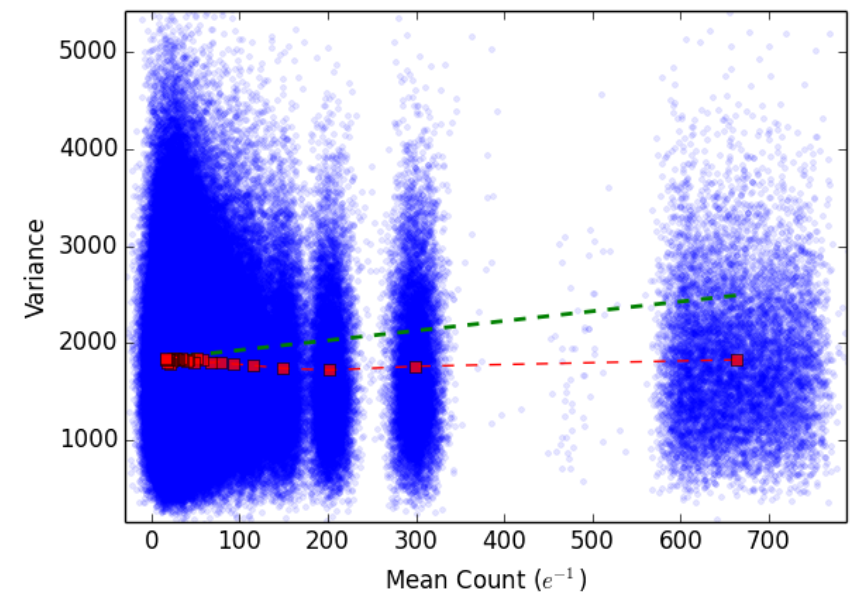

Fig. 8. (Color online) Variance versus mean counts. The green dashed line shows the expected increase if the counts are of Poissonian statistics like dark current, and the red squares represent the measured variance obtained by averaging of the blue points, each representing pixels in a $100 \times 100$ subarray with origin at $(400,400)$ in the full array.

variance. If the large counts obtained in the initial seconds of the exposure are due to dark current, we expect a Poisson noise variance in them. Figure 8 shows the counts versus the variance. Blue dots are points corresponding to pixels from a good $100 \times 100$ subarray of pixels with origin at $(400,400)$ of the full array. The red squares correspond to the mean of the blue points corresponding to each set of readouts. The plot shows that the red points are almost constant and do not increase linearly with the counts. The green dashed line shows the expected increase in variance if the counts had Poissonian statistics, as a function of the counts. This implies that the reset anomaly does not have any significant contribution in the final noise of the data if we just discard the first few readouts (see Sec. 2.4.1).

\subsection{Readout noise}

Readout noise mainly comes from the amplifier noise in the electronics circuit. These were found to be uncorrelated in our 0.9-s readout time interval of each pixel during an exposure. Since dark current was found to be very small compared to the readout noise after $27 \mathrm{~s}$ of integration, we used difference of consecutive frames in SUTR readout of a dark frame to estimate $\sqrt{2}$ times readout noise. The readout noise was found to be different for each quadrant. Figure 9 shows the histogram of readout noise for single readout of different pixels in the array. The mean readout noise for single readout for 


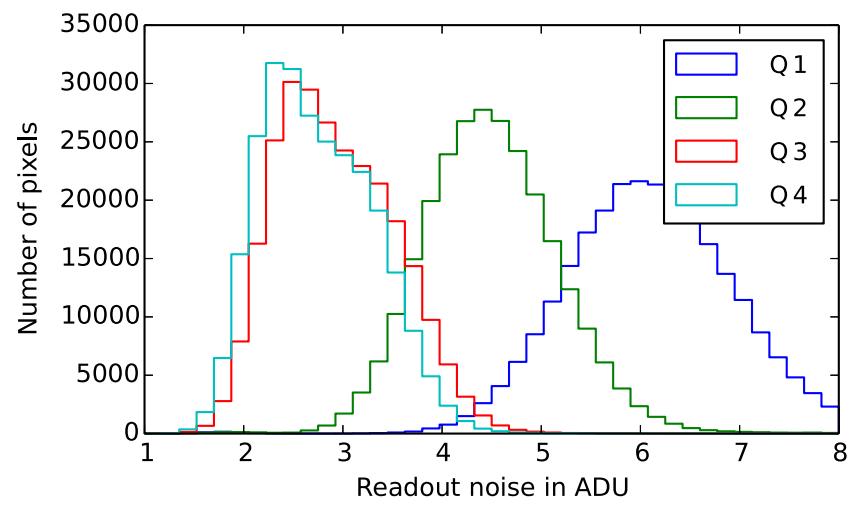

Fig. 9. (Color online) Histogram of readout noise for single readout in the array. The four histograms correspond to the four quadrants in the array.

each quadrant is $6.1,4.5,2.9$ and $2.8 \mathrm{ADU}$, with an average value of $4.1 \mathrm{ADU}$ (i.e. $\sim 25 e^{-}$) for the full array. It should be noted that this value is the readout noise for a single readout, hence readout noise contribution in the flux estimate is usually much less than this, and it is a function of the number of readouts in a single exposure and flux. Detailed treatment of error contribution of readout noise in flux calculation in SUTR (line-fitting) mode is available in Robberto (2007).

\subsection{Gain}

Gain is the multiplicative factor which converts ADU counts obtained from the detector array to electrons. This is crucial to estimate the actual photon counts obtained to estimate photon noise. For measuring the gain, we took multiple exposures of different exposure times with the array exposed to the telescope enclosure with the K filter. Since the thermal flux from the telescope is stable over the short duration of our exposures, these images are purely photon noise limited. For each pixel we calculated the slope of ADU flux counts versus variance. Since photon noise follows Poissonian statistics, actual electron counts versus variance will have a unity slope. Hence, the multiplicative gain factor was calculated by taking the inverse of the slope in ADU counts versus variance plot. Figure 10 shows the histogram of the gain obtained for each pixel in the array. The median gain of the detector was found to be $\sim 6 e^{-} / \mathrm{ADU}$.

\subsection{Saturation level (linear range)}

Unlike optical CCDs, NIR detectors have strong non-linearity near the saturation level of the pixels.

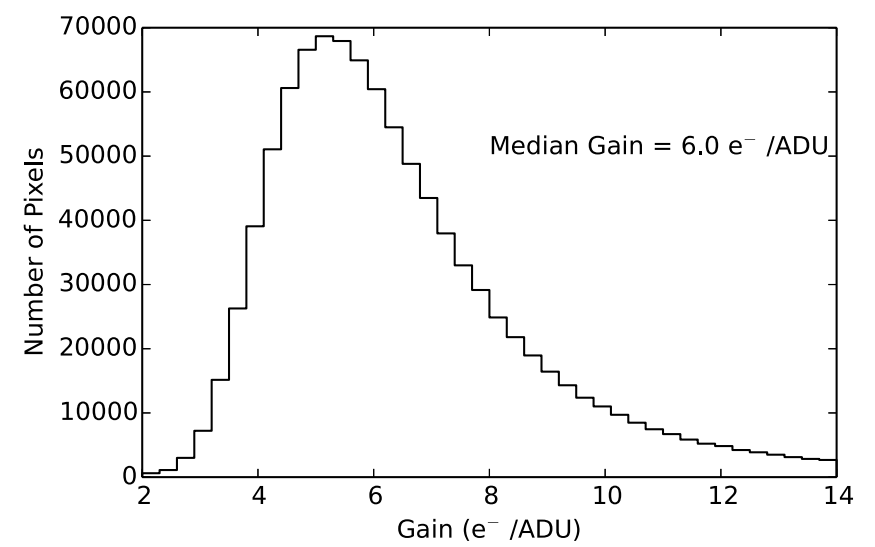

Fig. 10. The histogram of $\mathrm{ADU}$ to $e^{-}$gain of each pixel in the array.

Each pixel saturates and deviates from the linear regime at slightly different counts. Using the SUTR readout data, we estimated the upper limit of linear regime for each pixel separately. The median saturation point of the pixels was found to be at $\sim 16,000$ ADU, subtracting the median bias level of $\sim 5700$ ADU, gives us an effective well depth of $\sim 10,300$ ADU $\left(\sim 61,800 e^{-}\right)$. By default, all the data in the non-linear regime are discarded, but we have added a provision in the data reduction software for users to do non-linearity correction in the nonlinear regime and save the corrected data if needed (see Secs. 2.4.1 and 5).

\subsection{Bad, hot and cold pixels}

Bad pixels are defined to be those pixels in the array which deviate more than $8 \sigma$ from the median value in the image obtained by dividing two flats taken in high and low incident flux. This set includes all the pixels whose gain is varying with time or are completely dead. In our detector array, most of such pixels are along the edges. The percentage of the bad pixels in the array is $0.1 \%$. Apart from this, during some of the nights, the vertical single strip of pixels at column position 512 (at the border of quadrants) was also found to have a varying gain. Hence, we avoid objects falling on that column during our observations.

Hot pixels are defined to be pixels which have counts more than $8 \sigma$ above the median value in a dark readout of $100 \mathrm{~s}$. Their fraction was found to be $2.3 \%$ (most of them are located at the four quadrant corners heated by the glow from the nearby readout transistor). Similarly, cold pixels (pixels which have counts less than $8 \sigma$ below median value in dark) fraction was found to be $0.07 \%$. It is to be noted 
that, only a subset of cold and hot pixels are actually bad pixels, which need to be masked, and the remaining can be corrected by flat fielding and dark subtraction. For robustness of all the above calculations, standard deviation $\sigma$ was estimated from the median absolute deviation.

\subsection{Spectral resolving power}

The reciprocal linear dispersion of TIRSPEC in spectroscopy mode is $\sim 4.7 \AA /$ pixel. The effective spectral resolving power of the spectra in each order was estimated by finding full-width-half-maximum (FWHM) of argon lamp lines through the narrow slit of $0.9^{\prime \prime}$ width. Figure 11 shows the median $\lambda / \Delta \lambda$ spectral resolving power along the slit obtained from argon lines as a function of wavelength.

\subsection{Fringe issue}

Spectra in $\mathrm{K}$ band show fringes in the detector. HAWAII-1 PACE arrays are known to have this issue due to interference in the sapphire substrate on which the $\mathrm{HgCdTe}$ detector material is grown (Hodapp et al., 1996). Figure 12 shows the

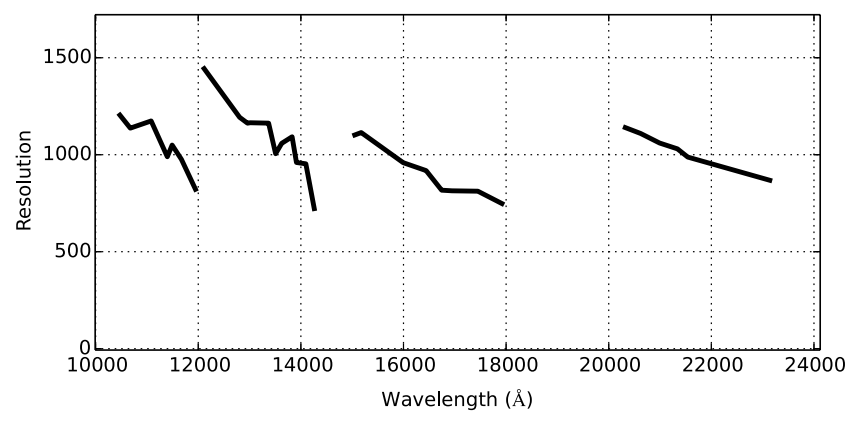

Fig. 11. Median spectral resolving power measured as a function of wavelength from argon lamp spectrum.

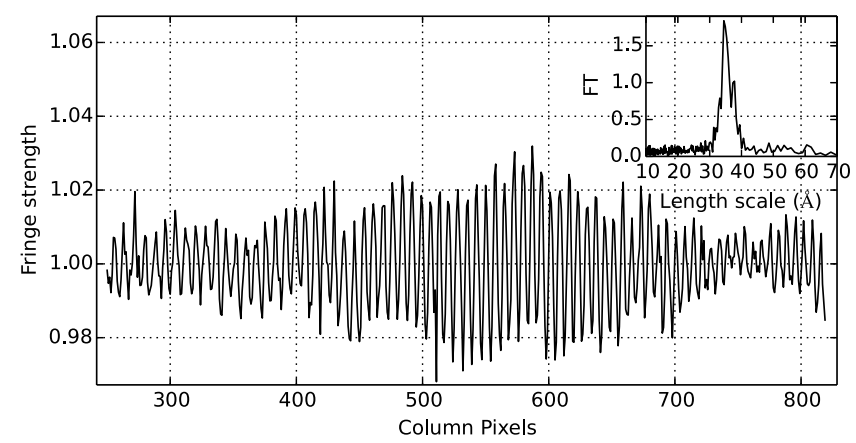

Fig. 12. Normalized fringe pattern seen in $\mathrm{K}$ band spectrum. Fourier transform of the fringe pattern (shown in inset) gave the spatial size of fringes to be $\sim 36 \AA$. normalized strength of fringes seen in $\mathrm{K}$ band of $0.9^{\prime \prime}$ slit spectrum. The fringe pattern was found to be stable and its Fourier transform gave a typical wavelength scale equivalent to $\sim 36 \AA$ in dispersed spectrum. Since this is close to our typical stellar line widths, it is not safe to use any Fourier transform based fringe removal technique. However, since the fringes are stable, it can be removed by normalizing with contemporaneous flat and standard star spectra taken at the same position along the slit.

\subsection{Persistence issue}

HAWAII-I PACE array detectors are known to have strong persistence and this leaves a slowly decaying persistence image if exposed to very bright light. The time scale for decay of persistence image is a function of the strength of bright light which caused persistence. A shift of 23 pixels perpendicular to the dispersion direction in the position of the spectrum by the grism in TIRSPEC avoids the spectrum from overlapping on the persistence of bright star image taken for centering the star in the slit.

\section{Performance on HCT}

\subsection{Plate scale and field of view}

We matched 2MASS coordinates of stars in the selected NIR standard star fields catalog (Hunt et al., 1998) to the pixel coordinates in TIRSPEC image. The WCS submodule in Astropy ${ }^{(d)}$ and optimize submodule in $\operatorname{Scipy}^{(\mathrm{e})}$ was used to fit the world coordinate parameters by least square minimization. The plate scale of TIRSPEC images obtained is 0.30 arcsec/pixel. With the measured plate scale, we obtain a FoV of $307 \times 307 \operatorname{arcsec}^{2}$ $\left(\sim 5 \times 5 \operatorname{arcmin}^{2}\right)$ for the $1024 \times 1024$ array.

\subsection{Sky background}

In NIR, where the sky is almost 800 times brighter than in the optical, the faint magnitude limit in the imaging mode is limited by photon noise from the sky background. To estimate the sky brightness at $\mathrm{HCT}$, we observed NIR standard star fields from Hunt et al. (1998) on various nights. The sky brightness in $\mathrm{H}$ and $\mathrm{K}$ bands

\footnotetext{
d Astropy Collaboration (2013).

${ }^{\text {e }}$ Scipy is a standard package for scientific computing with Python (Oliphant, 2007).
} 
was found to vary depending on the humidity during the nights. This is expected, since the major contribution of flux in the $\mathrm{H}$ band comes from water and $\mathrm{OH}$ telluric lines. The median values of sky brightness obtained in good night conditions are the following: $\mathrm{J}=16.14 \mathrm{mag} / \mathrm{arcsec}^{2}$, $\mathrm{H}=13.70 \mathrm{mag} / \operatorname{arcsec}^{2}, \mathrm{~K}_{\mathrm{s}}=13.35 \mathrm{mag} / \operatorname{arcsec}^{2}$ and $\mathrm{K}=13.20 \mathrm{mag} / \operatorname{arcsec}^{2}$. These are comparable to other good sites like Mauna Kea (4145 m amsl) and Paranal (2635 m amsl).

The other important related parameter is the time scale of variation in sky brightness. This determines how fast one should observe separate sky frames to subtract from object frames (especially while observing diffuse/extended emission regions). Time scales also vary considerably from night to night. To obtain typical time scales, we monitored the sky brightness of one region continuously in intervals of 17 seconds. Figure 13 shows the autocorrelation of sky brightness in the $\mathrm{J}, \mathrm{H}$ and $\mathrm{K}_{\mathrm{s}}$ filters. The e-folding time scale in auto-correlation is $\sim 600 \mathrm{~s}$ in the $\mathrm{H}$ and $\mathrm{K}_{\mathrm{s}}$ bands at $\mathrm{HCT}$ site, whereas the time scale is longer in the $\mathrm{J}$ band.

\subsection{Throughput of the entire system}

The throughput of the entire system was measured by counting the total flux from standard stars over an area of 4 times the FWHM of their profile. The image mode throughput percentage is $16 \pm 5 \%, 20 \pm 5 \%$ and $20 \pm 5 \%$ for the $\mathrm{J}, \mathrm{H}$ and $\mathrm{K}_{\mathrm{s}}$ bands respectively. This percentage corresponds to the net throughput and it includes the atmosphere, telescope mirrors, instrument optics and

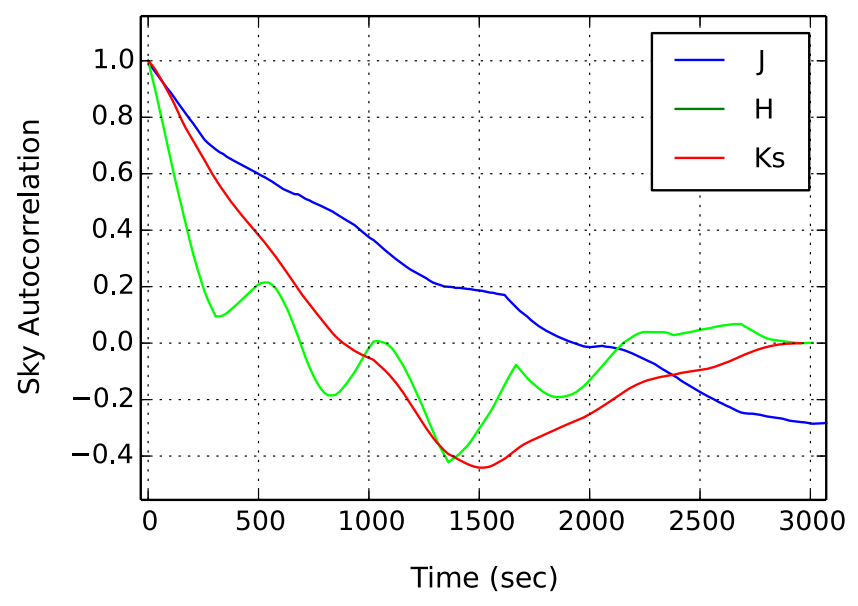

Fig. 13. (Color online) Blue, green and red curves show the auto-correlation of the $\mathrm{J}, \mathrm{H}$ and $\mathrm{K}_{\mathrm{s}}$ sky brightness respectively. The e-folding time scale gives the typical time scale in which sky brightness varies at the HCT site. quantum efficiency (QE) of the detector. These values are consistent with our initial theoretical estimate of throughput. Spectral throughput is difficult to measure by this method, because of the difficulty in placing the star at the exact center of the slit every time. However, we have a theoretical estimate of the spectral throughput to be $30 \%$, which does not include the QE of the detector and the slit losses.

While the cross dispersed mode of spectroscopy has the huge advantage of simultaneously taking two orders of spectrum and even slightly extended wavelength coverage at the $2.4-\mu \mathrm{m}$ end, there is a trade off in the net throughput between the single order and the cross dispersed modes due to the additional grism. Figure 14 shows the measured fractional throughput of the cross dispersed mode relative to the single order mode. This was obtained by taking the ratio of the continuum tungsten lamp spectra in the single and cross dispersed modes through the same slit.

\subsection{Photometric sensitivity}

The estimate of the limiting magnitude depends on the seeing conditions. During our instrument calibration nights, FWHM of the star profile was never below $1.4^{\prime \prime}$ in the $\mathrm{K}_{\mathrm{s}}$ band. To estimate the photometric sensitivity, we observed crowded standard star fields (Hunt et al., 1998) and obtained the estimated magnitude versus magnitude error plot. Figure 15 shows the $\mathrm{J}, \mathrm{H}$ and $\mathrm{K}_{\mathrm{s}}$ magnitudes versus their magnitude errors obtained for the sources in the AS 40 field (Hunt et al., 1998) for $170 \mathrm{~s}$ of exposure at $40^{\circ}$ elevation on a night with seeing of $1.8^{\prime \prime}$. The photometry was carried out with an aperture of radius $1 \mathrm{FWHM}$.

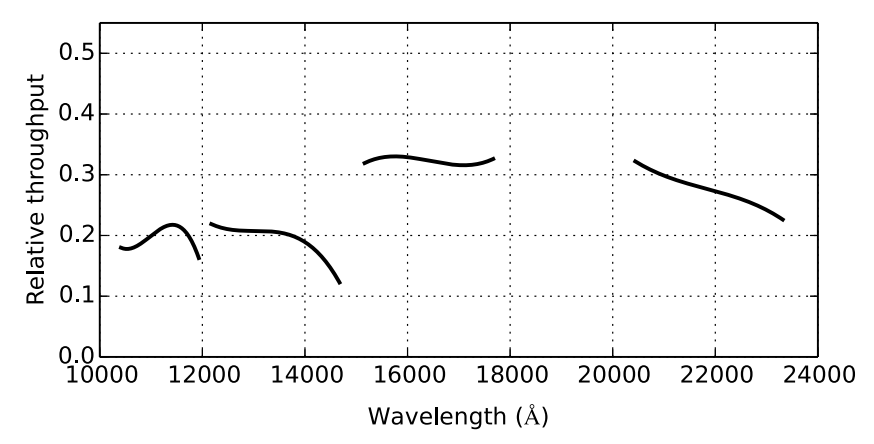

Fig. 14. Four curves showing throughput in the cross dispersed mode normalised with respect to the corresponding single order mode in each band. This fractional reduction in net throughput is due to the additional grism used in the cross dispersed mode. 


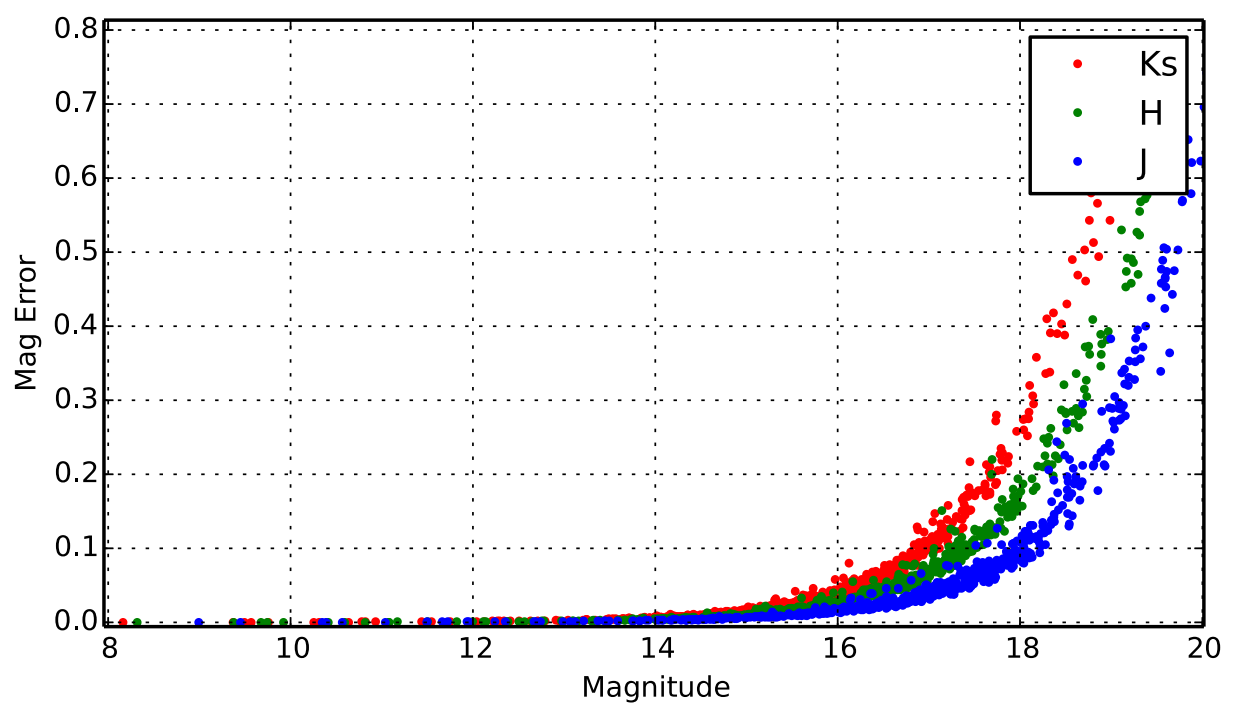

Fig. 15. J, H and $\mathrm{K}_{\mathrm{s}}$ magnitudes versus magnitude errors plot for the sources in the AS 40 field, taken with $170 \mathrm{~s}$ of total exposure. The photometry was carried out with an aperture of radius 1 FWHM.

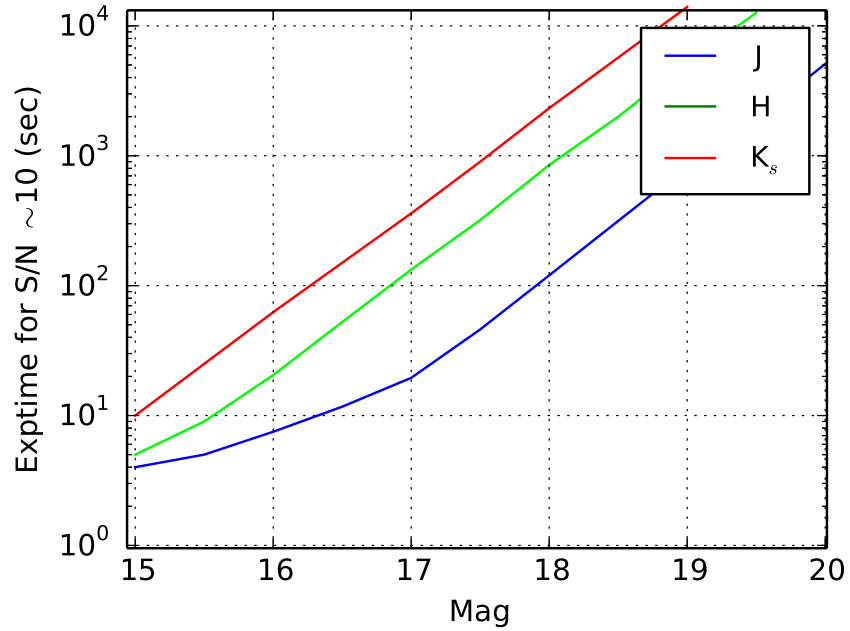

Fig. 16. Exposure time required for $0.1 \mathrm{mag}$ error (10\%) photometry versus star magnitudes in the $\mathrm{J}, \mathrm{H}$ and $\mathrm{K}_{\mathrm{s}}$ bands.

Figure 16 shows the exposure time required for $0.1 \mathrm{mag}$ error (10\%) photometry for various star magnitudes on a typical night. It is generated by calculating the signal-to-noise $(\mathrm{S} / \mathrm{N})$ ratio for slope fitting (Robberto, 2007) and integrating the entire $\mathrm{S} / \mathrm{N}$ ratio contribution for a given Gaussian star profile of $\mathrm{FWHM}=1.6^{\prime \prime}$ and photometric aperture of radius 1 FWHM.

\subsection{Spectroscopic sensitivity}

Attainable spectroscopic sensitivity for different magnitudes were estimated by taking spectra of faint stars. Figure 17 shows various estimates of exposure time required for a continuum $\mathrm{S} / \mathrm{N} \sim 10$

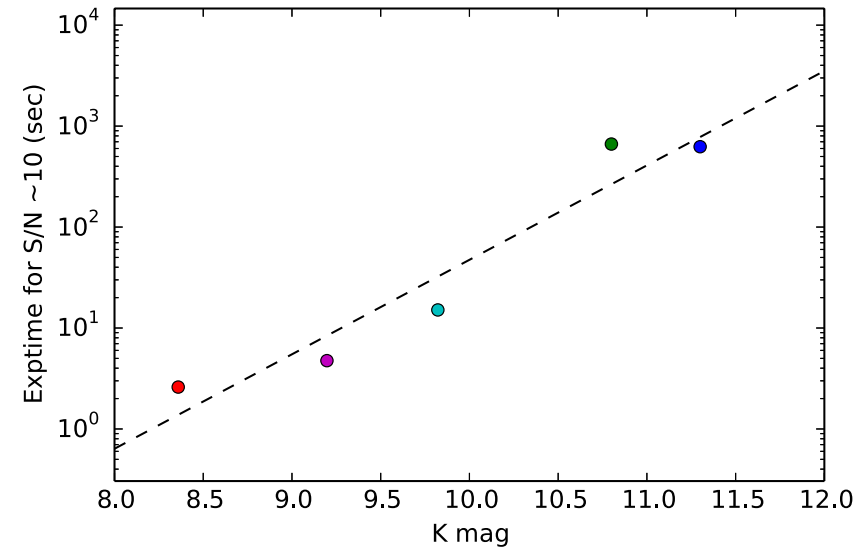

Fig. 17. Various estimates of exposure time required in single order mode for $\mathrm{S} / \mathrm{N} \sim 10$ in continuum in the $\mathrm{K}$ band versus $\mathrm{K}$ band magnitude of the source on typical nights observed from HCT site.

in single order mode. It is quite sensitive to the FWHM of the star profile as well as the accuracy in centering of the star inside the slit. We have found that spectra of stars upto $12 \mathrm{mag}$ in the $\mathrm{J}, \mathrm{H}$ and $\mathrm{K}$ bands can be obtained under typical (1.6" seeing) night conditions.

\subsection{Color equations}

For accurate $(1 \%)$ broad band photometry, we need to correct for the filter response with stars of different color. Coefficients of 1st order color equations were obtained by fitting a straight line to magnitude zero points of standard stars with a wide range of colors. To establish the color equations, 
we have observed the color ranges from 0.45 to 2.5 for $\mathrm{J}-\mathrm{K}_{\mathrm{s}}, 0.35$ to 1.76 for $\mathrm{J}-\mathrm{H}$, and 0.06 to 2.83 for $\mathrm{H}-\mathrm{K}_{\mathrm{s}}$. To improve the statistics, we simultaneously fitted the slopes of many standard star fields, letting the airmass correction term free to vary from one field to another. Standard star fields were taken from Hunt et al. (1998) and red standard stars from Persson et al. (1998). The color correction equations obtained for TIRSPEC at HCT site for calibrating to 2MASS magnitudes are the following.

$$
\begin{aligned}
J-K & =(0.97 \pm 0.03) *(j-k)+\beta_{J K} \\
K-k & =(0.11 \pm 0.03) *(J-K)+\beta_{K k} \\
J-H & =(1.00 \pm 0.05) *(j-h)+\beta_{J H} \\
J-j & =(0.12 \pm 0.04) *(J-H)+\beta_{J j} \\
H-K & =(0.82 \pm 0.07) *(h-k)+\beta_{H K} \\
K-k & =(0.07 \pm 0.03) *(H-K)+\beta_{K k}
\end{aligned}
$$

\subsection{Field of view distortions}

To measure any distortions in the FoV, we used the coordinates of 2MASS sources in a crowded field observed with TIRSPEC. After matching the coordinates of stars in the TIRSPEC image with the 2MASS field, we used the geomap tool in $\operatorname{IRAF}^{(f)}$ to obtain second order transformation coefficients to quantify FoV distortion. A third degree general

${ }^{\mathrm{f}}$ IRAF is distributed by the National Optical Astronomy Observatory, which is operated by the Association of Universities for Research in Astronomy, Inc., under cooperative agreement with the National Science Foundation.

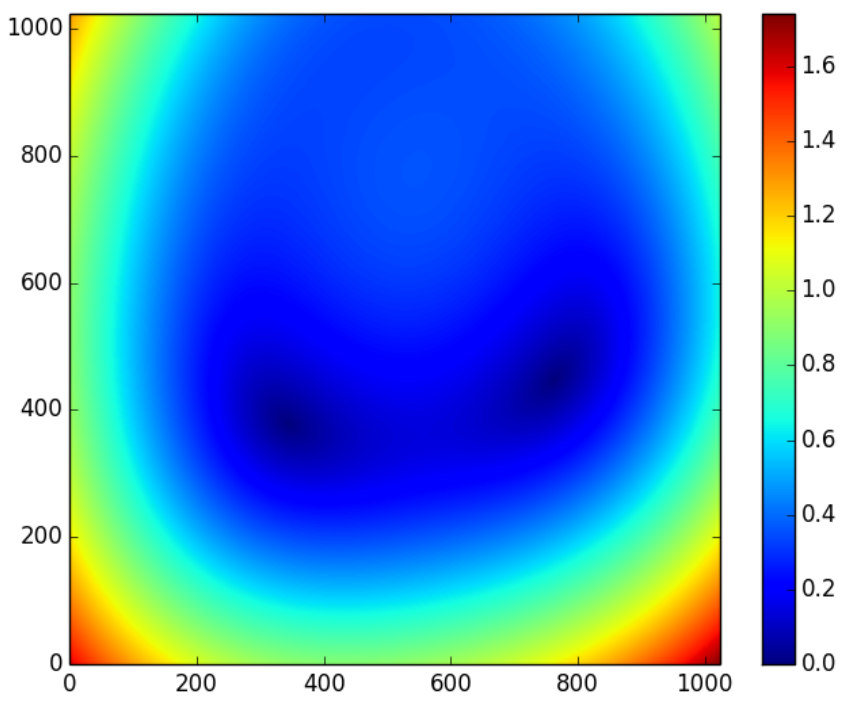

Fig. 18. (Color online) The color map shows the magnitude of deviation from the linear estimate of FoV in units of arcsec.

polynomial fit was also done to study the FoV distortions. Figure 18 shows the measured deviation from linear FoV in the array plane. The units are in arcsec. Significant FoV distortions are found only towards the edge of the array.

\subsection{Sample images and spectra}

During the engineering runs, to test the scientific capabilities of the instrument, we observed various astrophysical sources in imaging as well as in spectroscopy modes. The objects observed range from star-forming regions, transient sources like young low mass star eruptive variables, novae (Ninan et al., 2013b), supernovae (Srivastav et al., 2014)

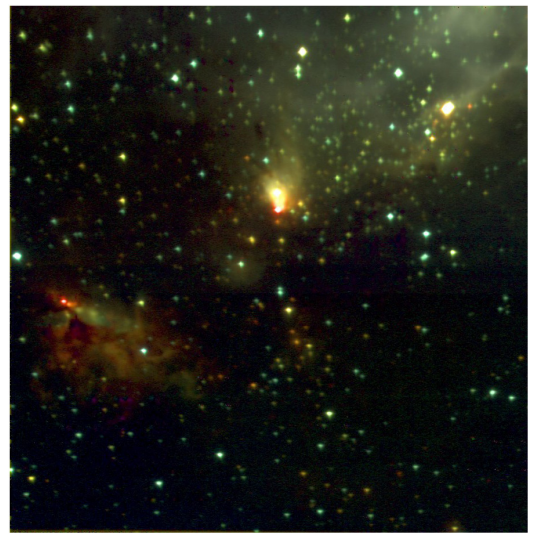

(a)

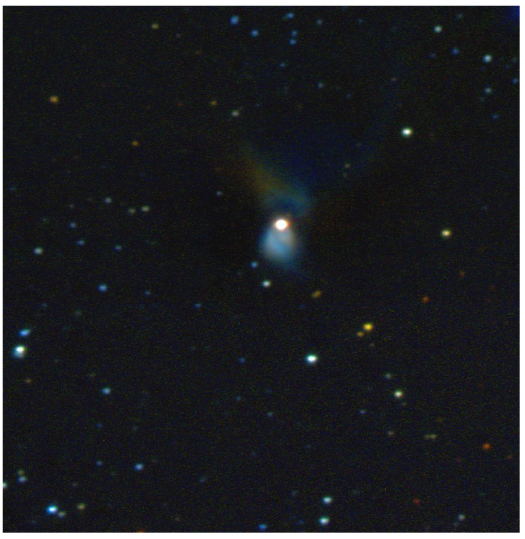

(b)

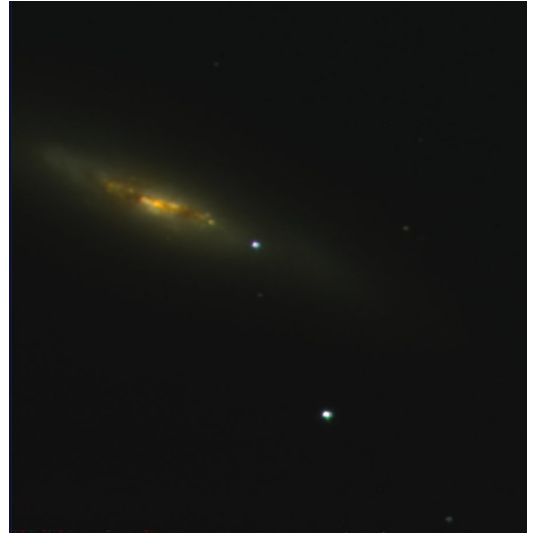

(c)

Fig. 19. (Color online) Various RGB color composite images generated using TIRSPEC J (blue), H (green) and $\mathrm{K}_{\mathrm{S}}$ (red) images. On the left is NGC 7538 (star-forming region), at the middle is V2494 Cyg (FU Ors family of outburst source), and on the right is SN2014J (Supernova in M82). 


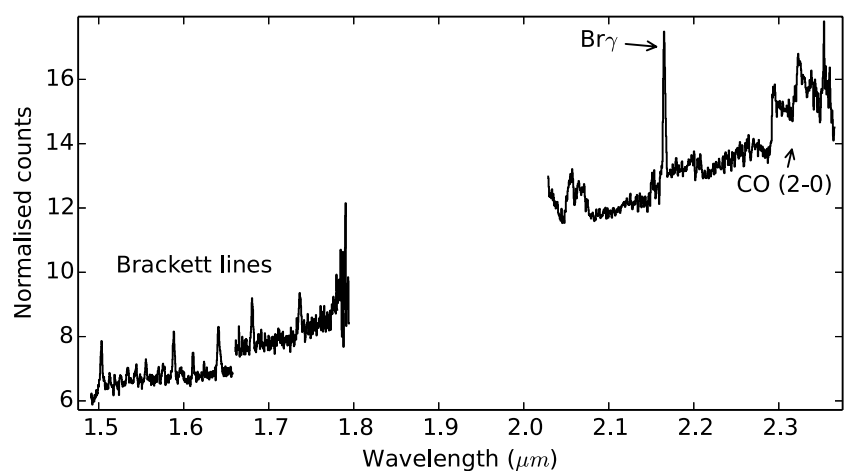

(a)

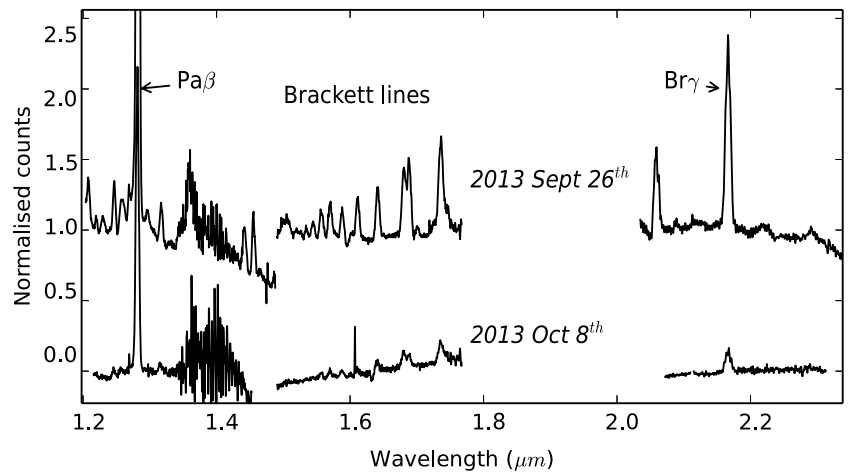

(c)

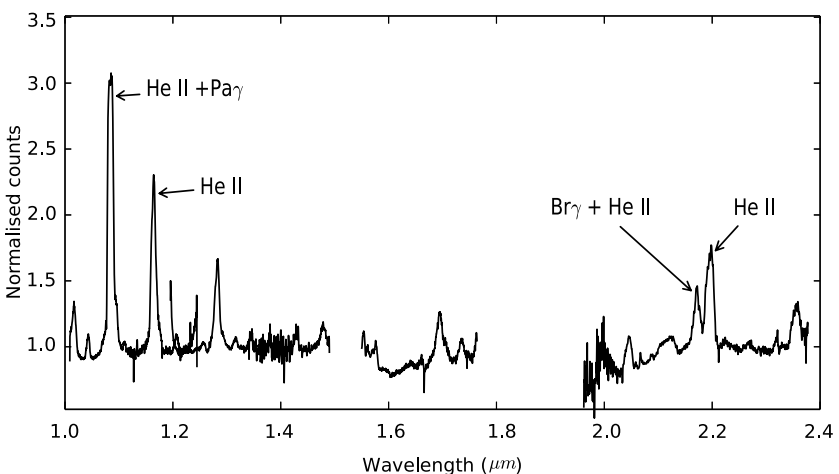

(b)

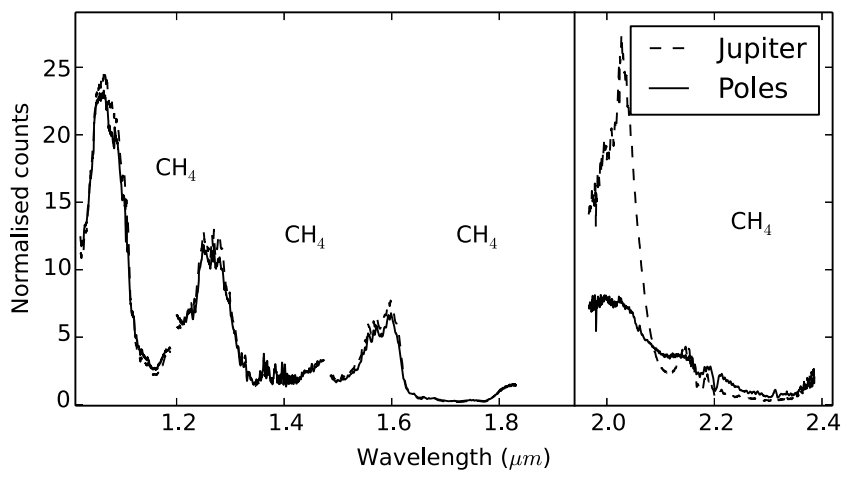

(d)

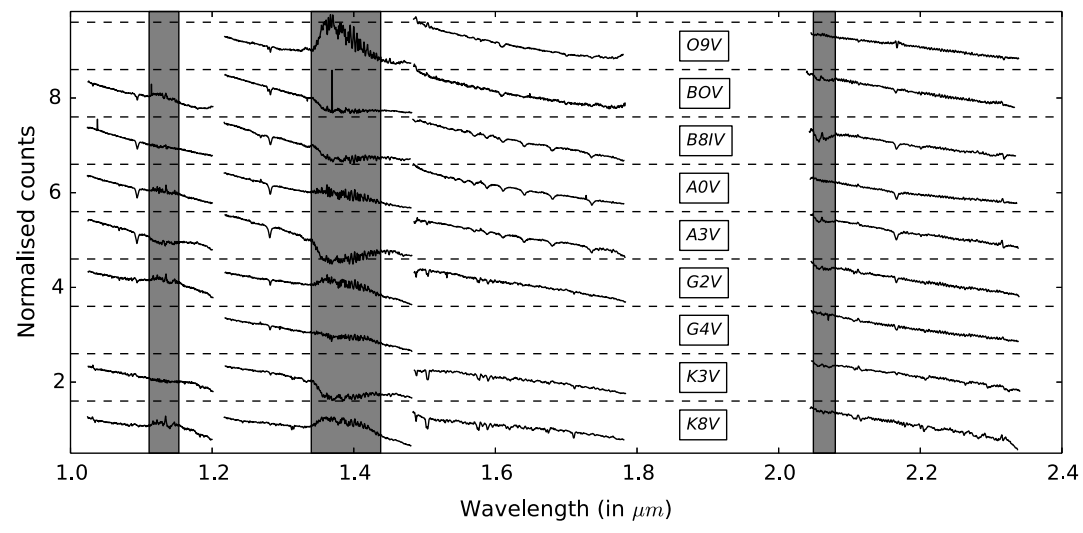

(e)

Fig. 20. Sample of the NIR spectra taken with TIRSPEC. (a) PV Cep (FU Ori kind of outburst source with outflow jets), (b) WR 6 (Wolf Rayet star), (c) Nova Del 2013 (NIR spectral evolution of nova), (d) Spectrum of Jupiter and its poles (K band spectrum is scaled for clarity), (e) Stellar spectra of various main-sequence spectral type stars.

and Wolf Rayet stars to objects like white dwarfs, our solar system planets, and stars from various positions in the main sequence of HertzsprungRussell diagram. Figure 19 shows color composite images created from the $\mathrm{J}, \mathrm{H}$ and $\mathrm{K}_{\mathrm{s}}$ images of some of the sources observed with TIRSPEC. Figure 20 shows the NIR spectra obtained from various kinds of sources taken with TIRSPEC.

\section{Data Reduction Tool}

We have developed the data reduction tool for analysis of TIRSPEC data with a philosophy to automate all the repetitive steps in data reduction, without any compromise on the quality of the reduced data. This semi-automated pipeline requires human guidance only in flagging good and 
bad frames, choosing sources of interest for photometry, etc. This tool is especially useful when many nights of data of a particular source have to be reduced together. Codes are written in Python, using standard modules used by the astronomy community like astropy, ${ }^{(\mathrm{g})}$ numpy, ${ }^{(\mathrm{h})}$ matplotlib $^{(\mathrm{i})}$ and PyRAF. ${ }^{(j)}$ All the codes are written in modular form with inbuilt documentation, so users can easily modify or create their own pipeline by importing the codes as modules. The latest version of codes (under GNU GPLv3 license) are regularly updated on the instrument website. ${ }^{(\mathrm{k})}$ The data reduction tool has mainly three parts as explained below.

\subsection{Slope image generation}

TIRSPEC and the telescope are controlled remotely by the observer from Centre for Research \& Education in Science \& Technology, Hosakote, IIA, Bangalore (India) via a satellite link. In one typical night of observations, the SUTR data acquisition mode in which TIRSPEC is operated can generate upto $50 \mathrm{~GB}$ of raw data. With the existing link bandwidth, it is not possible to download all the raw data to the control room in Bangalore on the same day. Hence, we generate the slope images at the HCT site itself. The first part of the data reduction tool, which does dark subtraction, intelligent pixel masking, and slope fitting (see Sec. 2.4.1) is run on the TIRSPEC computer at Hanle. This part is fully automated and run at the end of every observation night. The data that is received by the observer in the Bangalore control room are the dark-subtracted slope images. Two log files are generated and provided to the observer, one containing the $\log$ of the image generation pipeline, and the other containing a summary of all observations done during the night. The same code can be imported as a module in an interactive Python terminal if the user wants to work on the raw SUTR data.

\subsection{Photometry}

The photometric part of the TIRSPEC data reduction tool does basic reduction and contains

\footnotetext{
${ }^{\mathrm{g}}$ Astropy Collaboration (2013).

${ }^{\mathrm{h}} \mathrm{NumPy}$ is the fundamental package for scientific computing with Python (Oliphant, 2007).

${ }^{\mathrm{i}}$ Hunter (2007).

${ }^{\mathrm{j}} \mathrm{PyRAF}$ is a product of the Space Telescope Science Institute, which is operated by AURA for NASA.

${ }^{\mathrm{k}}$ http://web.tifr.res.in/daa/tirspec/.
}

options to do aperture photometry as well as PSF photometry using daophot IRAF binaries. In order to not compromise on the quality of data reduction, selection of good and bad frames is done by human supervision. The code also allows users to run the script in an interactive mode, which allows the user to verify the steps during the process of execution. Photometric magnitude outputs are written to a log file.

The $\mathrm{S} / \mathrm{N}$ ratio calculation in the SUTR readout is slightly more complicated than simple doublecorrelated readouts. We have made an exposure time calculator for TIRSPEC, which first creates a model star profile based on seeing conditions, star magnitude, sky brightness and zero point magnitude of TIRSPEC. The $\mathrm{S} / \mathrm{N}$ ratio for a set of aperture radii is then numerically calculated using gain, readout noise and number of non-destructive readouts in the exposure using the error propagation formula of the SUTR mode (see Robberto (2007) for the equations). Figure 21 shows the screenshot of this tool.

\subsection{Spectroscopy}

The spectroscopy part does basic reduction and finally produces 1D wavelength-calibrated spectrum. Human supervision is again needed for identifying the good frames to be reduced. It is also advisable to run this task in an interactive mode to cross check the automated argon line identification during wavelength calibration. Once the

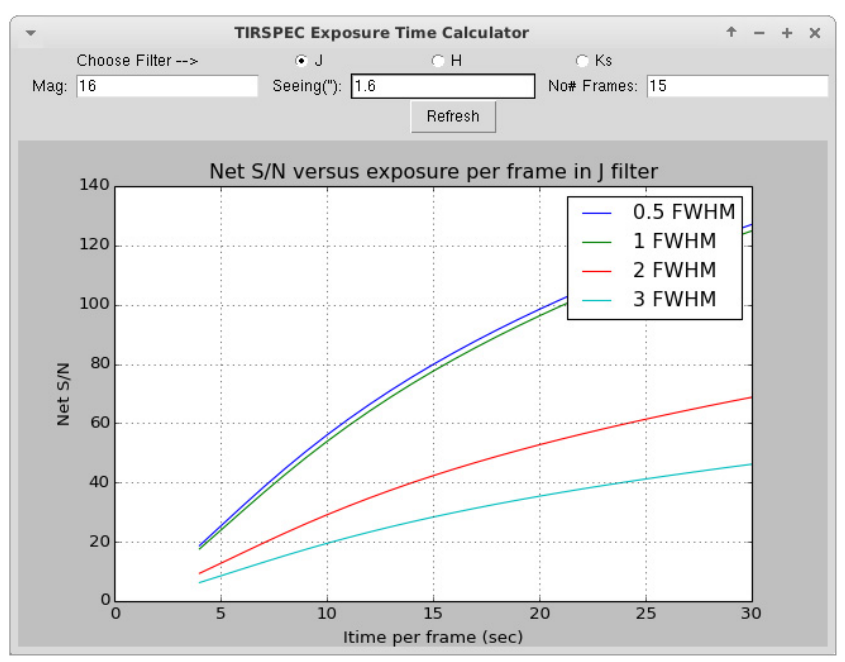

Fig. 21. (Color online) Screenshot of the $\mathrm{S} / \mathrm{N}$ ratio estimator. Various curves show the $\mathrm{S} / \mathrm{N}$ ratio that can be obtained for corresponding photometry aperture radius as a function of exposure time per frame. 
wavelength-calibrated spectra of the science target as well as a standard star are obtained, one can use the Telluric Correction tool to remove the telluric absorption lines in the spectra.

\subsubsection{Telluric Correction tool}

NIR spectra contain many telluric lines and their removal is done by dividing with the spectrum of a spectroscopic NIR standard star taken within the nearby airmass immediately before or after the target observation. Along with telluric lines, this also removes the instrument response and gives us a continuum-corrected spectra. This step also removes fringes in the $\mathrm{K}$ band spectra. The Telluric Correction tool is a fully interactive software which removes the standard star's intrinsic stellar lines, aligns and scales before dividing and finally applies black body temperature correction to obtain the continuum-corrected spectrum of the source.

\section{Conclusion}

This paper discusses the successful installation, characterization and engineering phases of the TIRSPEC. As seen from the results, the TIRSPEC mounted on HCT, Hanle, is quite a competitive NIR instrument which has opened up competitive NIR spectroscopy to the Indian astronomy community. After successful characterization and performance analysis runs on the telescope, TIRSPEC is now officially released to the astronomy community for science observations from May 1, 2014. It is currently being used heavily for science observations by various astronomers in India. In the near future we plan to change the $10^{\prime \prime}$ short slits to $50^{\prime \prime}$ slits of the same widths. This will allow for dithering of the star position along the slit while taking spectra. Subarray readout capability of the array will also be implemented soon, which will allow us to take much faster readouts of a small section of the array by compromising on the FoV.

Note added in proof: In July 2014, we upgraded all the $10^{\prime \prime}$ slits to $50^{\prime \prime}$ slits of the same widths.

\section{Acknowledgments}

We thank referee Dr. Rainer Lenzen for giving us invaluable comments and suggestions that improved the content of the paper. The authors would like to thank the staff at the HCT, Hanle, for their co-operation during the installation and characterization and daily maintenance of the TIRSPEC. We especially thank Mr. Angchuk Dorje, Mr. Tsewang Dorjai and Mr. Sonam Jorphail who made crucial contributions during the installation phase. We would also like to thank the staff at CREST and especially Prof. B. C. Bhatt and Prof. D. K. Sahu, Hosakote, for their enthusiasm and help during all the observation runs with TIRSPEC.

\section{References}

Acosta-Pulido, J., Dominguez-Tagle, C. \& Manchado, A. [2003] Proc. SPIE 4841, 437.

Finger, G., Mehrgan, H., Meyer, M. et al. [2000] Proc. SPIE 4008, 1280 .

Ghosh, S. K. [2010] ASI Conference Series 1, 167.

Ghosh, S. K., Iyengar, K. V. K., Rengarajan et al. [1988] ApJ 330, 928 .

Hodapp, K. W., Hora, J. L., Hall, D. N. B. et al. [1996] New Astronomy 1(2), 177.

Hunt, L. K., Mannucci, F., Testi, L. et al. [1998] AJ 115(6), 2594.

Hunter, J. D. [2007] Computing in Science \& Engineering $\mathbf{9}(3), 90$.

Naik, M. B., Ojha, D. K., Ghosh, S. K. et al. [2012] Bull. Astr. Soc. India 40, 531.

Ninan, J. P., Ojha, D. K., Bhatt, B. C. et al. [2013a] ApJ $\mathbf{7 7 8}(2), 116$.

Ninan, J. P., Ojha, D. K., Ghosh, S. K. et al. [2013b] ATel \#5269.

Ojha, D. K., Ghosh, S. K., D'Costa, S. L. A. et al. [2012a] Astr. Soc. India Conference Series 4, 189.

Ojha, D. K., Ghosh, S. K., D'Costa, S. L. A. et al. [2012b] Astr. Soc. India Conference Series 4, 191.

Oliphant, T. E. [2007] Computing in Science 8 Engineering $\mathbf{9}(3), 10$.

Persson, S. E., Murphy, D. C., Krzeminski, W., Roth, M. \& Rieke, M. J. [1998] AJ 116(5), 2475.

Rieke, G. H. [2007] ARAESA 45(1), 77.

Riopel, M., Doyon, R., Nadeau, D. \& Marois, C. [2004] in Scientific Detectors for Astronomy, Amico, P., Beletic, J. W. \& Beletic, J. E. (eds.) (Springer, Netherlands), p. 453.

Robberto, M. [2007] Instrument Science Report WFC3 200712, http://adsabs.harvard.edu/abs/2007wfc..rept...12R.

Robitaille, T. P., Tollerud, E. J., Greenfield, P. et al. (Astropy Collaboration) [2013] $A \mathscr{E} A$ 558, 33.

Srivastav, S., Ninan, J. P., Anupama, G. C., Sahu, D. K. \& Ojha, D. K. [2014] ATel \#5876. 